\title{
An Efficient Trademark Image Retrieval using Combination of Shape Descriptor and Salience Features
}

\author{
Saurabh Agarwal ${ }^{1}$, Nikhil Chaturvedi ${ }^{2}$ and Punit Kumar Johari ${ }^{3}$ \\ ${ }^{1,2,3}$ Department of CSE/IT, M.I.T.S., Gwalior \\ 1'im100rav@gmail.com, ${ }^{2}$ er.nikhil.chaturvedi@gmail.com, \\ ${ }^{3}$ pkjohari@mitsgwalior.in
}

\begin{abstract}
Trademark carries the prestigious values for a particular company so it is very important to distinguish it from the similar context trademark. In this research paper, we propose an efficient Trademark Image Retrieval (TIR) model which is a branch of Content Based Image Retrieval (CBIR). In the proposed system we extract the edge point of a particular image and after this the edge point are evaluated to find the corner pixel from it. For the performance evaluation of the system we use the most commonly used method namely precision-recall. From the experimental result we conclude that the TIR based on shape feature perform better and gives satisfactory result.
\end{abstract}

Keywords: Content Based Image Retrieval, Trademark Image Retrieval, Edge Detection, Corner Point Detection

\section{Introduction}

In past few years number of image collections available has increased due to ease of capturing images by different acquisition systems. The storage format of image data is relatively standardized however the effective retrieval of images from such databases remains a significant challenge. Content-based image retrieval (CBIR) [1] is an emerging research topic for multimedia databases and digital libraries. CBIR was introduced by Kato in a workshop on visual information management system organized in National Science Foundation of United States. There is a growing interest on CBIR from academia, industry, Government, Non-Government agencies, and other Organizations. There are four important feature components for content-based image retrieval: color, texture, shape, and spatial relationship. Among these features, shape contains the most attractive visual information for human perception. Shape representation [2] compared to other features, like texture [3, 15] and color [4, 12], is much more effective in semantically characterizing the content of an image. The construction of shape descriptors is even more complicated when invariance, with respect to a number of possible transformations, such as scaling, shifting and rotation, is required [5].

The CBIR System is based on the features extracted from the images. Figure 1. shows the CBIR based shape image retrieval model. 


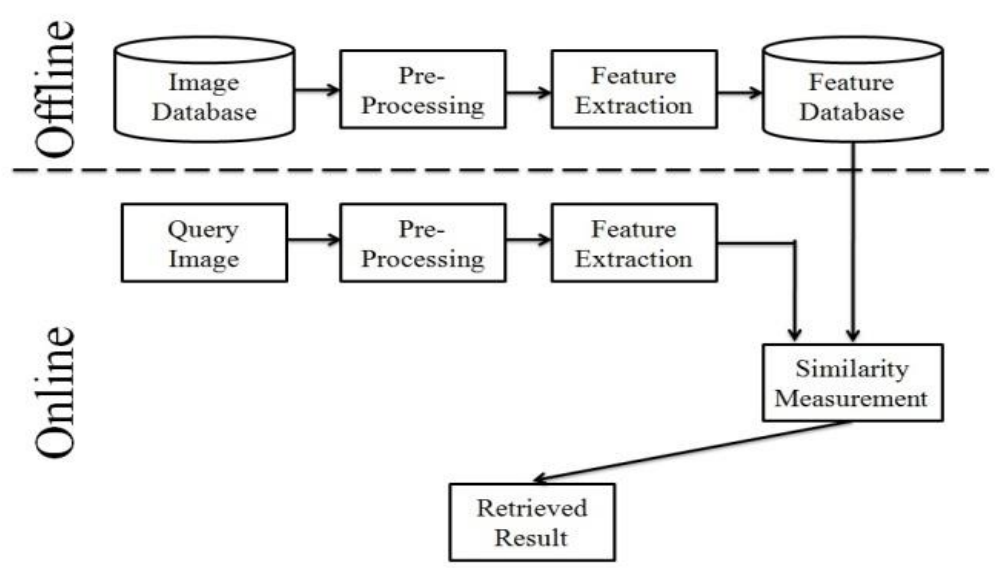

Figure 1. Image Retrieval Model

As a branch of Shape based CBIR, the research of trademark image retrieval (TIR) [6] is of great practical significance. For example if a company wants to register a new trademark, they must find whether there is any similar trademark in existing database. Existing trademarks retrieval is mainly based on manual classification code [7]. With the increase of registered trademarks by human becomes laborious. Trademarks can be categorized into few different types [8], a trademark image retrieval system can only be designed to accommodate one of the types. Although several trademark retrieval systems have designed to handle all kinds of trademark images, the performance of these systems are rather unfavourable when compared to those systems specifically designed to handle only one kind of trademark. Human perception for similarity between trademark images is also another challenge for trademark image retrieval system.

\subsection{Shape Descriptor}

Shape is a very important part of an image; it contains some basic visual features for describing the image contents. Shape can use information extracted from various manners like shape contour features or features from whole shape. Various shape descriptors exist in the literature, mainly categorized into two groups:

a. Contour-based shape descriptors.

b. Region-based shape descriptor.

Contour-based shape descriptors $[9,10]$ need extraction of boundary information. Boundary detection and corner extraction is useful in contour-based shape descriptor. Region-based shape descriptors do not relay on boundary information, but they take into account all the pixels within the shape region.

1.1.1. Edge Detection: Edge detection is an important process in most of the image understanding systems. The overall performance of these systems depends on accuracy and reliability of edge detection method. Sobel Operation and Prompt Edge Detection Method are the most simple and efficient method for edge detection. The Sobel operation uses four masks to detect edge points. The Prompt edge detector detects edge points by checking the differences in the gray value for each point from those of its neighbours. In the experiment we find that as the image size increases the efficiency of the prompt edge detection is found better as compared to Sobel. 


\section{Proposed Methodology}

In our proposed work, we initially created a feature database by extracting the feature points of the database images. These feature points are edge based corner features. After creating the dataset the query image is accepted by the retrieval system. The same feature points are generated for the query image. At last the similarity measurement function is applied on the feature points which results all the relevant images from the image database.

The shape feature used in the flow of proposed retrieval system is discussed below: -
a. Prompt Edge Detection.
b. Corner Point detection.

\subsection{Prompt Edge Detection}

In this method the edge is detected using the 8 - connected neighbours. In this the difference between the gray level values of the neighbouring pixel are calculated to find that weather the desired pixel is edge point or not.

The pseudo code for prompt edge detection [13] is shown below:

1. Select the input image I.

2. Find the image size in row an column form

$$
[R, C]=\operatorname{size}(I) \text {; }
$$

3. For each pixel in the image, Repeat step 4 to 6

4. Calculate the absolute difference between all the 8 neighbouring pixels.

5. Find the number of difference that exceeds the local threshold (T).

$$
\begin{aligned}
& \text { If, } \quad \text { difference }>\mathrm{T} \\
& \text { Then, } \mathrm{k}(\text { difference count })=\mathrm{k}+1
\end{aligned}
$$

6. If, $3<\mathrm{k}<6$

Then, the above pixel is an edge pixel.

7. Connect all the calculated edge pixels in a single image to obtain the desired result.

\subsection{Corner Point Detection}

Corner point [14] detection is an approach to detect certain feature point which has a regional maximum value as compared to their neighbouring pixels. At corner point the pixels have frequent changes in the intensity values. These frequent changes can be use to judge the corner points.

The pseudo code for Corner point detection is shown below:

1. Select the input image I.

2. Generate the corner metric matrix of the image I.

$$
\text { CM=cornermetric (I); }
$$

3. Find the corner peaks in the CM matrix.

$$
(x, y)=\text { Corner Index. }
$$

4. Plot all the corner coordinates in the image.

5. Calculate the total no. of corner in the image. 


\subsection{Threshold Function}

According to our experimental results and corner values stored in the database, we conclude that as the number of corner increases in the image the difference between their relevant images corner values also increases.

\section{So, Threshold $\propto$ Corner Count}

\section{Proposed Algorithm}

To retrieve the relevant images from the database we use the Shape salience features i.e. the corner point detection which is applied on the edge pixel images. The working algorithm for the above methodology is discussed below:

1. Select the input image I.

$$
I=\text { Query image }
$$

2. Convert the image in gray scale intensity values.

$$
\text { Input_image =rgb2gray }(I) \text {; }
$$

3. Find the Edge pixel image using Prompt edge detection.

$$
\text { Edge_image=Prompt_edge (Input_image); }
$$

4. Find the corner points of the Edge pixel image.

$$
\text { Corner_count }=\text { corner_point }(\text { Edge_image }) \text {; }
$$

5. Apply the similarity measurement algorithm

$$
\text { Difference_value }=\mid \text { Corner_count-Corner_count_database } \mid
$$

6. Display the images from the database that occurs in minimum and maximum threshold region.

The flow chart of the working model is shown in Figure 2.



Figure 2. Flow Chart for the Proposed Image Retrieval System 


\section{Experimental Results}

\subsection{Trademark Dataset}

In our experiment we have taken an approx of 108 trademark images for testing our algorithm. The Trademark Dataset [16] is not deliberately designed; the images are selected from the MPEG 7 Standard Dataset. The dataset is designed in such a manner, so that our algorithm will get structural challenges like rotational changes. The Trademark dataset Contains 18 different classes of images, having each class image to be rotated in 5 different angles i.e. $60^{\circ}, 120^{\circ}, 180^{\circ}, 240^{\circ}, 300^{\circ}$.

\subsection{Experiment Setup}

In our proposed work, the main goal is to develop such an image retrieval system which matches the human perception. The result obtained using proposed algorithm is shown in the Table 1. The precision-recall technique is used to study the evaluated results. The precision-recall is a very common technique used in Trademark image retrieval process for performance evaluation of the system. In experiment, the values of precision and recall are calculated using following formulae:

$$
\begin{aligned}
& \text { precision }=\frac{n_{r}}{T_{r}} \\
& \text { recall }=\frac{n_{r}}{T_{s}}
\end{aligned}
$$

Where,

$\mathrm{n}_{\mathrm{r}}=$ Number of similar images in the retrieved result.

$\mathrm{T}_{\mathrm{r}}=$ Total number of images in the retrieved result.

$\mathrm{T}_{\mathrm{s}}=$ Total number of similar images in the database.

Table 1. Retrieved Result and their Precision-Recall Value

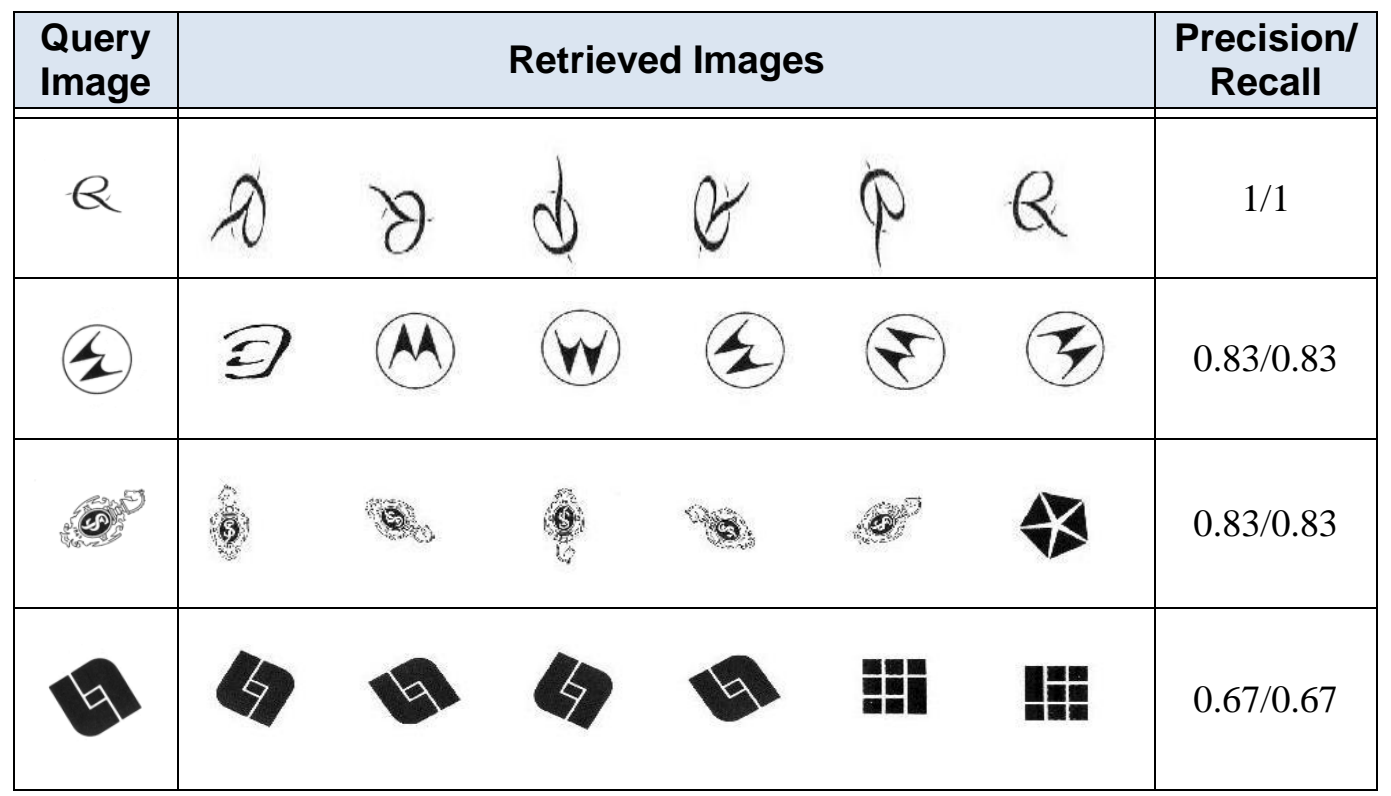




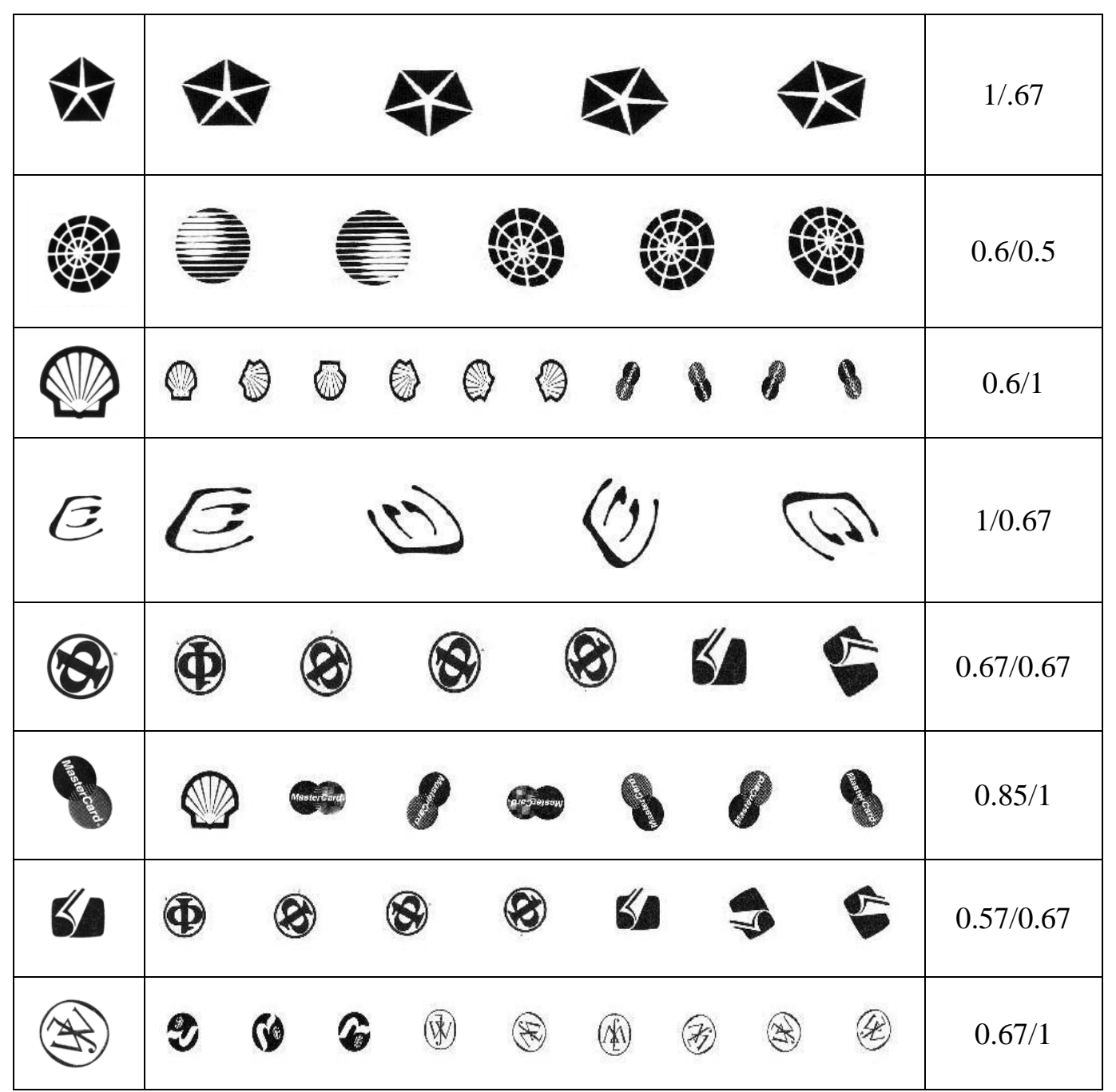

\section{Conclusion and Future Work}

In this paper, we have studied different types of trademarks having transformational changes. We have restricted our work to only handle the rotational challenges, but in future we are planning for further experimentation with more challenging dataset and by having more accurate algorithms. In this work, we have combined retrieval algorithms based on edge detection and corner detection, and the results are satisfactory. In future we propose more accurate algorithms to get better results by the help of clustering techniques and with the help of image filtering process to attain our desired result.

\section{Acknowledgements}

The authors would like to thank the anonymous reviewers for their constructive comments.

\section{References}

[1] Kato, T. (1992). Database architecture for content-based image retrieval. Image Storage and Retrieval Systems, Proc SPIE 1662, 112-123.

[2] Song, J., Lu, X., Ling, H., Wang, X. \& Tang, Z. (2012). Envelop Extraction for composite shapes for shape retrieval. ICPR, 1932-1935. 
[3] Agrawal, D., Jalal, A.S. \& Tripathi, R. (2013). Trademark Image Retrieval by Integrating Shape with Texture Feature. IEEE, 30-33.

[4] Cheng, Y.F. \& Cong, Z. (2010). The Technique of Color and Shape-based multi- Feature Combination of Trademark image Retrieval. IEEE .

[5] Belongie, S., Malik, J. \& Puzicha, J. (2002). Shape Matching and object Recognition using Shape contexts. IEEE Trans., 502-522.

[6] Qi, H., Li, K., Shen, Y. \& Qu, W. (2010). An effective solution for trademark image retrieval by combining shape description and feature matching. Elsevier, 2017-2027.

[7] Schietse, J., Eakins, J.P. \& Veltkamp, R.C. (2007). Practice and Challenges in Trademark Image Retrieval. CIVR.

[8] Anuar, F.M., Setchi, R. \& Lai, Y.K. (2012). Trademark image Retrieval using integrated shape descriptor. Elsevier.

[9] Amanatiadis, A., Kaburlasos, V.G., Gasteratos, A. \& Papadakis, S.E. (2011). Evaluation of shape descriptors for shape-based image retrieval. IET Image Process, 493-499.

[10] Arafat, S.Y., Saleem, M. \& Hussain, S.A. (2009). Comparative Analysis of Invariant Schemas for Logo Classification. IEEE, 256-261.

[11] Wei, C.H., Li, Y., Chau, W.Y. \& Li, C.T. (2008). Trademark Image Retrieval Using Synthetic features for describing global shape and interior structure. Elsevier, 386-394.

[12] Wang, Y.J., Chun \& Zheng, F. (2009). Trademark Image Retrieval Based on Shape and key Local Color Features. IEEE, 325-328.

[13] Lin, H.J., Kao, Y.T., Yen, S.H. \& Wang, C.J. (2004). A Study of shape based Image Retrieval. IEEE, 118-123.

[14] Pedrosa, G.V., Batista, M.A. \& Barcelos, C.A.Z. (2013). Image feature Descriptor based on shape salience points. Elsevier, 156-163.

[15] Chaturvedi, N., Agarwal, S. \& Johari, P.K. (2014). A Novel approach of image retrieval based on Texture. IJERMCA , 42-48.

[16] Logo Database for Research

[17] http://lampsrv02.umiacs.umd.edu/projdb/project.php?id=47

\section{Authors}

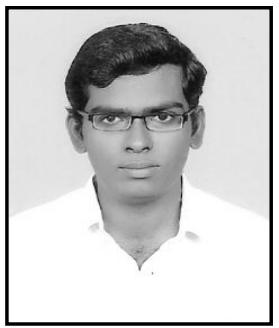

Saurabh Agarwal, male, is currently an M.Tech. Student at Madhav Institute of Technology and Science, Gwalior, India. He got his bachelor degree from Laxmi Narayan Institute of Technology, Gwalior, India in 2012. His research interest includes digital image processing and pattern recognition.

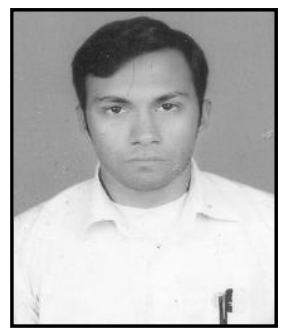

Nikhil Chaturvedi, male, is currently an M.Tech. Student at Madhav Institute of Technology and Science, Gwalior, India. He got his bachelor degree from Mahrana Pratap Engineering College, Kanpur, India in 2011. His research interest includes digital image processing and pattern recognition.

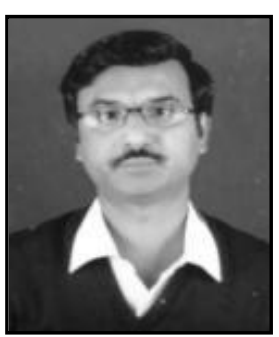

Punit Kumar Johari, male, is an Assistant Professor at Madhav Institute of Technology and Science, Gwalior, India. He has a working experience of 10 years in different colleges. His research interest includes Digital image processing, Pattern recognition and Data mining. 
International Journal of Signal Processing, Image Processing and Pattern Recognition Vol.7, No.4 (2014) 